\title{
CrystEngComm
}

Check for updates

Cite this: CrystEngComm, 2018, 20 6834

Received 23rd March 2018, Accepted 1st August 2018

DOI: 10.1039/c8ce00452h

rsc.li/crystengcomm

\section{Crystallisation of sodium dodecyl sulfate-water micellar solutions with structurally similar additives: counterion variation $\uparrow$}

\author{
Ruhina M. Miller, (iD a João T. Cabral, (1D ${ }^{b}$ Eric S. J. Robles, (1D ${ }^{c}$ \\ Nicholas J. Brooks ${ }^{a}$ and Oscar Ces ${ }^{\star a}$
}

\begin{abstract}
The effects of a series of structurally similar sodium dodecyl sulfate (SDS) additives on the crystallisation of SDS-water micellar solutions were investigated using a combination of differential scanning calorimetry, dynamic light scattering, optical microscopy and inductively coupled plasma optical emission spectroscopy. Seven different counterions were chosen from groups 1 and 2 of the periodic table to replace the sodium on SDS: LDS, (SDS), KDS, RbDS, CsDS, $\mathrm{Mg}(\mathrm{DS})_{2}, \mathrm{Ca}(\mathrm{DS})_{2}$ and $\mathrm{Sr}(\mathrm{DS})_{2}$. Two representative temperature profiles were employed - linear cooling ramps at rate of $0.5^{\circ} \mathrm{C} \mathrm{min}{ }^{-1}$ to determine near-equilibrium kinetics and transitions and isothermal holds at $6{ }^{\circ} \mathrm{C}$ to elucidate morphological changes. Crystallisation of the reference solution $20 \% \mathrm{SDS}-\mathrm{H}_{2} \mathrm{O}$ with $0.25,1.0$ and $2.5 \%$ additive was generally promoted or inhibited even at the lowest concentrations. Melting points however remained largely unchanged, suggesting that the additives predominantly had a kinetic rather than thermodynamic effect. ICP-OES measurements for the solutions containing $1 \%$ additive indicated that most of the additives were integrated into the SDS crystals which was reflected by morphological changes, including the formation of hexagonal and oval shaped crystals. Our results both quantify and provide a morphological insight into the effect of a series of additives on the crystallisation of micellar SDS solutions, which can readily form due to preferential Na exchange.
\end{abstract}

\section{Introduction}

Surfactants are frequently used in formulations for household and personal care products, pharmaceuticals, paints and textiles. $^{1-3}$ These are often derived from oleochemical and petrochemical sources for instance lard, palm and crude oils. ${ }^{2-4}$ Surfactants produced from these sources tend to have variability in their structures for example a mixture of chain lengths, ${ }^{3}$ and may contain impurities from the manufacturing process such as by-products from synthesis ${ }^{5}$ or hydrolysis reactions. Impurities can have potent effects on a surfactant's properties including changing its Krafft point, ${ }^{6}$ surface tension, ${ }^{7}$ and critical micelle concentration (cmc) values. ${ }^{8}$ These are also problematic for crystallisation processes as they can impact transition temperatures, the metastable zone width

\footnotetext{
${ }^{a}$ Department of Chemistry and Institute of Chemical Biology-CDT, UK. E-mail: o.ces@imperial.ac.uk

${ }^{b}$ Department of Chemical Engineering, Imperial College London, London SW7 $2 A Z, U K$

${ }^{c}$ The Procter \& Gamble Company, Newcastle Innovation Centre, Newcastle-UponTyne NE12 9TS, UK

$\dagger$ Electronic supplementary information (ESI) available: ICP-OES details including wavelengths and calculations, DLS and additional optical microscopy data. See DOI: 10.1039/c8ce00452h
}

(MSZW) and crystal habit, ${ }^{9-14}$ potentially reducing a solution's stability window or altering its flow and filtration properties.

Impurities or additives are also used to alter crystallisation kinetics or the crystal morphology ${ }^{15-17}$ often tailor-made or structurally-similar additives are utilised for this due to their ease of incorporation at crystal faces. ${ }^{10}$ Additives can work via a number of routes - inhibiting, promoting or having no effect on crystallisation. ${ }^{9,18}$ If an additive preferentially adsorbs onto a specific face it can inhibit its growth rate ${ }^{18-20}$ alternatively it might affect nucleation by lowering the supersaturation of the system ${ }^{10}$ or by acting as a seed crystal, ${ }^{18,20}$ thus increasing or decreasing crystallisation induction times $\left(t_{\text {ind }}\right)$ respectively. Additionally, additives may inhibit the rate of interconversion of one crystal form to another. ${ }^{16}$

Sodium dodecyl sulfate (SDS) also referred to as sodium lauryl sulfate (SLS) is an anionic surfactant utilised in a range of industrial and academic applications including detergents, ${ }^{1,21,22}$ stabilisation of emulsions ${ }^{23}$ and microfluidic droplets. ${ }^{24}$ The structure of SDS, the dodecyl sulfate moiety and the partial phase diagram of $\operatorname{SDS}^{6,25}$ are provided in Fig. 1(a)-(c) respectively. The crystallisation of SDS has been studied using a variety of techniques including optical microscopy (OM), ${ }^{26,27}$ differential scanning calorimetry $(\mathrm{DSC})^{25,27}$ and X-ray diffraction $(\mathrm{XRD})^{26,28-33}$ to elucidate structures and crystallisation kinetics. Although the other 
(a)

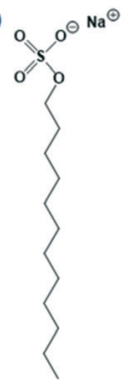

$20 \%$

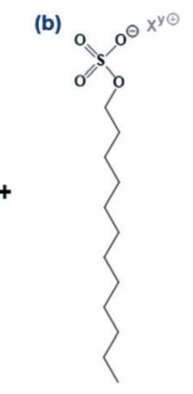

$0.25, \underline{1.0}, 2.5 \%$ (c)

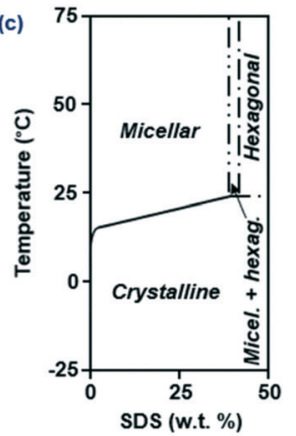

where $\mathrm{x}^{\mathrm{y}^{+}}=\mathrm{Li}^{+}, \mathrm{Na}^{+}, \mathrm{K}^{+}$, $\mathrm{Rb}^{+}, \mathrm{Cs}^{+} ; \mathrm{Mg}^{2+}, \mathrm{Ca}^{2+}, \mathrm{Sr}^{2+}$

Fig. 1 (a) Chemical structure of a SDS molecule; $20 \%$ was the baseline quantity used for all solutions. (b) $0.25-2.5 \%$ of an additive structurally comparable to SDS on which only the counterion " $X^{y+}$ " was varied. (c) Partial phase diagram of SDS- $\mathrm{H}_{2} \mathrm{O}$ (adapted from ref. 6 and 25); the aim of the study was to determine whether crystallisation and the phase boundary were affected by the presence of additives.

dodecyl sulfate crystals have received less attention, single crystal structures ${ }^{34,35}$ and Fourier transform infrared spectroscopy (FTIR) data ${ }^{36}$ are available for RbDS and $\mathrm{Mg}(\mathrm{DS})_{2}$, in addition to transition temperatures for KDS and RbDS micellar solutions. ${ }^{37}$ For additive studies in particular, SDS has been used as an additive to promote interconversion reactions ${ }^{38}$ and tune crystal morphologies, ${ }^{39-43}$ however the effects of additives (for example 1-dodecanol) on its crystallisation have only recently received attention. ${ }^{44}$ Possible impurities in the SDS system include 1-dodecanol if the product hydrolyses ${ }^{4,46}$ or other dodecyl salts from counterion exchange of the sodium, for example $\mathrm{Ca}(\mathrm{DS})_{2}$ in hard-water supplies.

We investigate the role of $0.25,1.0$ (primary focus) and $2.5 \%$ structurally similar SDS additives on the crystallisation kinetics of $20 \%$ SDS- $\mathrm{H}_{2} \mathrm{O}$ solutions under isothermal and linear cooling conditions. A wide range of additives was covered based on the substitution of the sodium ion - four counterions $\left(\mathrm{Li}^{+},\left(\mathrm{Na}^{+}\right), \mathrm{K}^{+}, \mathrm{Rb}^{+}, \mathrm{Cs}^{+}\right)$for the monovalent group 1 and three $\left(\mathrm{Mg}^{2+}, \mathrm{Ca}^{2+}, \mathrm{Sr}^{2+}\right)$ for the divalent group 2, as shown in Fig. 1. DSC at a fixed rate $(\phi)=0.5{ }^{\circ} \mathrm{C} \mathrm{min}^{-1}$ determined whether the additives promoted or inhibited crystallisation and affected the melting point, whilst dynamic light scattering (DLS) at stepwise temperatures ascertained whether any additional solubility boundaries were present. Lastly OM data at $T=6{ }^{\circ} \mathrm{C}$ provided morphological and kinetic comparisons, with inductively coupled plasma optical emission spectroscopy (ICP-OES) establishing whether the additive was integrated into the crystal. Results were compared to the reference solution where applicable, which we previously conducted isothermal $^{27}$ and linear cooling ${ }^{47}$ studies on.

\section{Experimental}

\section{Materials and methods}

SDS (ACS reagent grade, $\geq 99.0 \%$ purity, Sigma-Aldrich) was recrystallised from ethanol and dried under vacuum at $40{ }^{\circ} \mathrm{C}$ for 48 h. $^{25}$ LDS (supplier: VWR, manufacturer: Alfa Aesar, $\geq 99.0 \%$ purity) was used as received. The remaining metal dodecyl sulfate salts ("MDS" = KDS, RbDS, CsDS, Mg(DS) $\mathrm{Ca}(\mathrm{DS})_{2}, \operatorname{Sr}(\mathrm{DS})_{2}$ ) were synthesised from the corresponding metal chlorides ("MCl") ${ }^{48,49}$ by adding a saturated solution of $\mathrm{MCl}(15 \mathrm{ml})$ to a $10 \%$ solution of $\mathrm{SDS}-\mathrm{H}_{2} \mathrm{O}(5 \mathrm{ml})$; product details in the ESI† (Table S1). The solution was stirred for 15 min, filtered under vacuum, washed with $\mathrm{MCl}(5 \mathrm{ml})$ and $\mathrm{H}_{2} \mathrm{O}(5 \mathrm{ml})$ to yield a white powder, then dried under vacuum overnight. The powder was subsequently recrystallised from water and dried under vacuum for $48 \mathrm{~h}$. The product was characterised by inductively coupled plasma optical emission spectroscopy (ICP-OES) to determine the sodium to metal content and ${ }^{1} \mathrm{H}$ NMR spectroscopy to confirm the precipitate had a dodecyl sulfate unit.

Samples were prepared by weight percentage (wt\%), with $20 \mathrm{wt} \%$ SDS selected as the basis of these studies. To this $0.25,1.0$ or $2.5 \mathrm{wt} \%$ of MDS or "additive" was added to give a total SDS + MDS concentration of $20.25,21.0$ or $22.5 \%$, with deionised $\mathrm{H}_{2} \mathrm{O}$ accounting for the remaining 79.75-77.5 wt\%. An additional sample free of additives, namely 20\% SDS- $\mathrm{H}_{2} \mathrm{O}$ was also prepared as a reference. The samples were stirred until full dissolution then equilibrated for $\geq 24 \mathrm{~h}$ at $22{ }^{\circ} \mathrm{C}$; the solutions containing $2.5 \% \mathrm{Ca}(\mathrm{DS})_{2}$ and $0.25-2.5 \% \mathrm{Sr}(\mathrm{DS})_{2}$ required heating before use as the additive did not fully dissolve. Solutions were used for no longer than one week as SDS undergoes hydrolysis or no longer than one day for those requiring heating as this process is accelerated. ${ }^{45,46}$ Henceforth the solutions will generally be referred to by their additive only, for example $1 \%$ KDS or $1 \%$ solution(s).

\section{Differential scanning calorimetry (DSC)}

Thermal analysis was carried out on all sample compositions: 0.25-2.5\% MDS using a heat flux DSC (TA Instruments Q2000, $\pm 0.1{ }^{\circ} \mathrm{C}$ accuracy), calibrated with indium. Samples were filtered through $0.2 \mu \mathrm{m}$ PTFE syringe filters with $\approx 10 \mathrm{mg}$ sealed into hermetic aluminium pans (Tzero, codes: $901683.901 \&$ 901684.901) prior to each run. Samples were equilibrated for $10 \mathrm{~min}$ at $23{ }^{\circ} \mathrm{C}\left(\leq 25{ }^{\circ} \mathrm{C}\right.$ for $\mathrm{Ca}(\mathrm{DS})_{2}$ and $\leq 35{ }^{\circ} \mathrm{C}$ for $\left.\mathrm{Sr}(\mathrm{DS})_{2}\right)$ then cooled at $\phi=0.5{ }^{\circ} \mathrm{C} \min ^{-1}$ to $-5{ }^{\circ} \mathrm{C}$. The $20 \%$ SDS $-\mathrm{H}_{2} \mathrm{O}$ reference and the samples containing 1\% MDS were subsequently reheated at $\phi=0.5{ }^{\circ} \mathrm{C} \mathrm{min}^{-1}$ to determine the melting point. A minimum of two repetitions were conducted per composition with fresh samples used for each run; additionally the sample weights were recorded before and after each run to confirm that no mass was lost. Data was extracted using TA Instruments Universal Analysis 2000 software.

\section{Optical microscopy (OM)}

OM was conducted on the $1 \%$ MDS solutions. Solutions were filtered through $0.2 \mu \mathrm{m}$ PTFE syringe filters and were transferred into rectangular, flat capillary tubes (CM Scientific, cross-section $50 \times 500 \mu^{2}$ ). These were sealed with an epoxy-based adhesive (Araldite Rapid) and were left overnight to dry. This procedure was slightly modified for $1 \% \operatorname{Sr}(\mathrm{DS})_{2}$ 
as it precipitated at room temperature $\left(\approx 20{ }^{\circ} \mathrm{C}\right)$ - the sample was heated to $40{ }^{\circ} \mathrm{C}$, sealed with Parafilm $\mathrm{M}$, adhesive and equilibrated at $T=35{ }^{\circ} \mathrm{C}$ for $20 \mathrm{~min}$. For every experiment new capillaries were used to minimise the number of seed crystals and to reduce concentration variations due to repeated temperature cycling; the use of capillaries also allowed for rapid temperature equilibration. Three to four replicates were carried out per additive.

A reflection microscope (Olympus BX41M-LED, 10× objective) and temperature-controlled stage (Linkam Scientific THMS600) that has a stability and accuracy of $\pm 0.1{ }^{\circ} \mathrm{C}$ were used for the microscopy studies. Images were captured with a CCD camera (Allied Vision, Prosilica GX 1050C) and were examined using ImageJ $1.49 \mathrm{v}(\mathrm{NIH})$. Samples were equilibrated for $20 \mathrm{~min}$ at $22{ }^{\circ} \mathrm{C}$ (except for $1 \% \mathrm{Sr}(\mathrm{DS})_{2}$ detailed above), cooled rapidly at $50{ }^{\circ} \mathrm{C} \mathrm{min}^{-1}$ to $T=6{ }^{\circ} \mathrm{C}(t=0 \mathrm{~s})$ and held there until crystallisation was complete, specifically when no further growth was observed. At least three repetitions were acquired per additive.

\section{Dynamic light scattering (DLS)}

DLS was conducted on a Malvern Zetasizer Nano S (model: Zen 1600) for the 1\% MDS solutions, which was used as a turbidimeter to determine a (second) solubility boundary where applicable. Solutions were filtered and sealed in disposable PMMA cuvettes (VWR), equilibrated in a water bath for $20 \mathrm{~min}$ at $22^{\circ} \mathrm{C}$ and cooled at $\phi=0.5{ }^{\circ} \mathrm{C} \min ^{-1}$ to $-5{ }^{\circ} \mathrm{C}$. Afterwards the exterior of the cuvettes were cleaned and equilibrated in the DLS holder for $20 \mathrm{~min}$ at $20^{\circ} \mathrm{C}$. To determine the mean count rate, three measurements each averaging eleven runs were acquired every $1(0.5){ }^{\circ} \mathrm{C}$ until $30(37)^{\circ} \mathrm{C}$ (exception: $1 \% \operatorname{Sr}(\mathrm{DS})_{2}$ ), with a $120 \mathrm{~s}$ equilibration step per temperature point, scattering angle $\theta=173^{\circ}$. The data were collected using Zetasizer Software 7.01.

\section{Inductively coupled plasma optical emission spectroscopy (ICP-OES)}

ICP-OES was carried out on a PerkinElmer (OPTIMA 2000 DV) to determine the concentration of counterions $\left(\mathrm{M}^{+}\right)$in solution and subsequently the concentration of MDS or SDS. Standards were required for calibration therefore ICP multielement standard solution IV (Merck Millipore) was used for samples containing $\mathrm{Na}$ with $\mathrm{Li}, \mathrm{K}, \mathrm{Mg}$, $\mathrm{Ca}$ or $\mathrm{Sr}$, or the relevant metal chlorides for $\mathrm{Na}$ with $\mathrm{Rb}$ or Cs. For Cs a second set of standards containing $0.1 \% \mathrm{~K}^{+}\left[\mathrm{Cl}^{-}\right]$was also prepared to reduce ionisation interference which improved the signal. Both the standards and the samples were prepared in $\mathrm{a} \approx 2 \%$ $\mathrm{HNO}_{3}$ solution to reduce precipitation and to matrix match.

A blank and three standards (0.2, 5 and 25 ppm (25 and 50 ppm for Cs)) were initially run, with a higher concentration chosen for Cs due to the increased formation of ionic species. These results were corrected on the software (WinLab32) to ensure that the peak maximum and integration area were correctly selected for each element, wavelength and for the calibration curve. The samples could then be analysed for their $\mathrm{Na}$ and additive cation ("M", for example Li) concentrations, with three measurements acquired per sample. For the Cs samples containing $\mathrm{K}$ no overlaps were present in the Cs and $\mathrm{K}$ peaks. Further information including the wavelengths chosen are detailed in the ESI $\dagger$ (Table S2).

The synthesised MDS compounds were analysed for their $\mathrm{Na}$ and " $\mathrm{M}$ " content to determine the yield of MDS after counterion exchange; $\geq 99 \%$ was the target therefore the compound was recrystallised if this was not achieved. The product was dissolved in deionised water $(10 \mathrm{ml})$ to give a concentra-

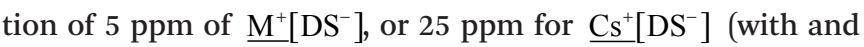
without $0.1 \% \mathrm{~K}^{+}$).

To determine whether any morphological changes observed via OM were due to integration of the additive into the crystal the $1 \%$ MDS solutions were selected. The initial solution $(10 \mu \mathrm{L})$ was diluted with deionised water $(10 \mathrm{ml})$ to serve as another ICP reference point. The remaining solution was then crystallised at $T=6^{\circ} \mathrm{C}$ between $1-3 \mathrm{~h}$ (based on OM data) via one of two methods: (1) in a syringe attached to a $0.2 \mu \mathrm{m}$ PTFE syringe filter, with the supernatant collected at $6{ }^{\circ} \mathrm{C}$ or (2) in an Eppendorf tube, which was centrifuged at $1700 \mathrm{rpm}$ for $5 \mathrm{~min}$ at $6^{\circ} \mathrm{C}$ before pipetting off the top layer of supernatant. For both methods the collected supernatant $(30 \mu \mathrm{L})$ was prepared for ICP in deionised water $(10 \mathrm{ml})$. The higher volume taken from the supernatant improved the sensitivity as a reduction in ion concentrations were expected. Each method was repeated twice for every counterion and the results are compared below. The calculations for the dilutions are provided in the ESI.†

\section{Results and discussion}

\section{DSC: overview}

DSC was used to investigate whether the additives acted as promoters or inhibitors of crystallisation based on shifts in the induction temperature $\left(T_{\text {ind }}\right)$ of $20 \%$ SDS- $\mathrm{H}_{2} \mathrm{O}$ solutions. Three additive concentrations: $0.25,1.0$ and $2.5 \%$ were studied. Samples were cooled between 23 (unless stated otherwise in the experimental section) and $-5{ }^{\circ} \mathrm{C}$ at $\phi=0.5{ }^{\circ} \mathrm{C} \mathrm{min}^{-1}$, as this rate allowed for reasonable temperature equilibration.

Fig. 2(a) shows a series of raw traces for $20 \%$ SDS- $\mathrm{H}_{2} \mathrm{O}$, $0.25,1.0$ and $2.5 \%$ KDS solutions, from which the onset $\left(T_{\text {ind }}\right)$ and peak $\left(T_{\mathrm{p}}\right)$ temperatures were extracted. ${ }^{50}$ DSC traces for the 1\% MDS solutions which are the primary focus of the study are provided in Fig. 2b(i) and (ii) for the group 1 and 2 counterions respectively, with the $20 \% \mathrm{SDS}-\mathrm{H}_{2} \mathrm{O}$ reference solution given as a dotted, black line. All profiles exhibited a single exothermic crystallisation peak. From these curves is apparent that going down group $1 T_{\mathrm{p}}$ initially shifts towards the phase boundary of $20 \%$ SDS- $\mathrm{H}_{2} \mathrm{O}\left(\approx 19.1^{\circ} \mathrm{C}\right),{ }^{47}$ therefore crystallisation is increasingly favoured. At KDS $T_{\mathrm{p}}$ reaches a maximum as a further increase in the ionic radius moves $T_{\mathrm{p}}$ back to lower values. For group $2 T_{\mathrm{p}}$ shifts to higher temperatures going down the group, with a sharper increase noted for $1 \% \operatorname{Sr}(\mathrm{DS})_{2}$. Both $T_{\text {ind }}$ and $T_{\mathrm{p}}$ show comparable trends, however $T_{\text {ind }}$ was preferred for 

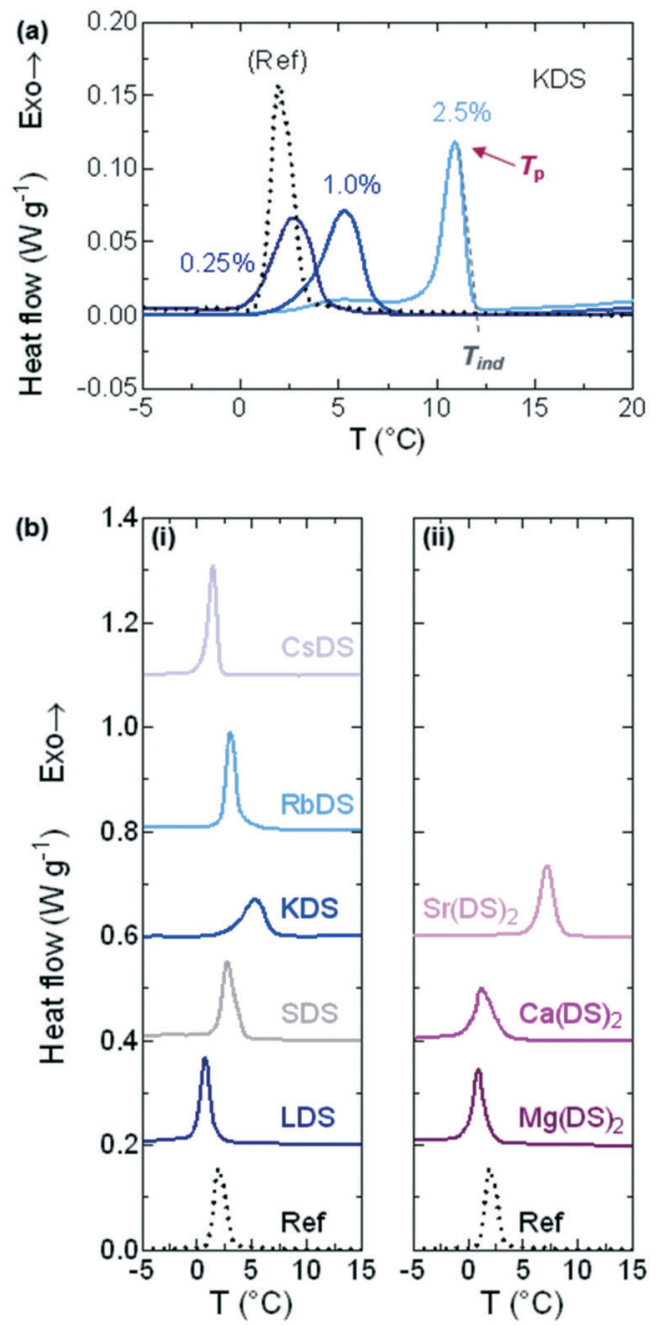

Fig. 2 DSC traces from 20\% SDS solutions spiked with additives (MDS), cooled linearly at $\phi=0.5{ }^{\circ} \mathrm{C} \mathrm{min}-1$ from the micellar phase. (a) Traces for the reference solution (20\% SDS- $\left.\mathrm{H}_{2} \mathrm{O}\right), 0.25,1.0$ and $2.5 \% \mathrm{KDS}$; from this the induction $\left(T_{\text {ind }}\right)$ and peak $\left(T_{\mathrm{p}}\right)$ temperatures were extracted. (b) Traces for the solutions containing 1\% additives for (i) group 1 and (ii) group 2 counterions. The dotted line is the reference solution with traces shifted in increments of 0.2 ( 0.3 for $\mathrm{Cs}$ ) W g $\mathrm{g}^{-1}$ for clarity.

analysis as this is when crystallisation begins and it is less affected by peak broadening.

\section{DSC: cooling ramps}

For all additive concentrations $T_{\text {ind }}$ was plotted against concentration, as shown in Fig. 3(a) and (b) for the group 1 and 2 counterions respectively. The flat, dotted black line represents the average $T_{\text {ind }} \approx 3.1{ }^{\circ} \mathrm{C}$ for $20 \%$ SDS- $\mathrm{H}_{2} \mathrm{O}$, whilst the grey curve on both graphs represents additional quantities of SDS. The effect of investigating this as an additive is twofold - it allows for the full trend to be established along group 1 and for the effects of additional dodecyl sulfate chains to be compared, essentially serving as a second set of reference measurements (in addition to $20 \%$ SDS $-\mathrm{H}_{2} \mathrm{O}$ ).

From these graphs it is evident that well-defined trends are observed for all counterions, with each acting as inhibi-
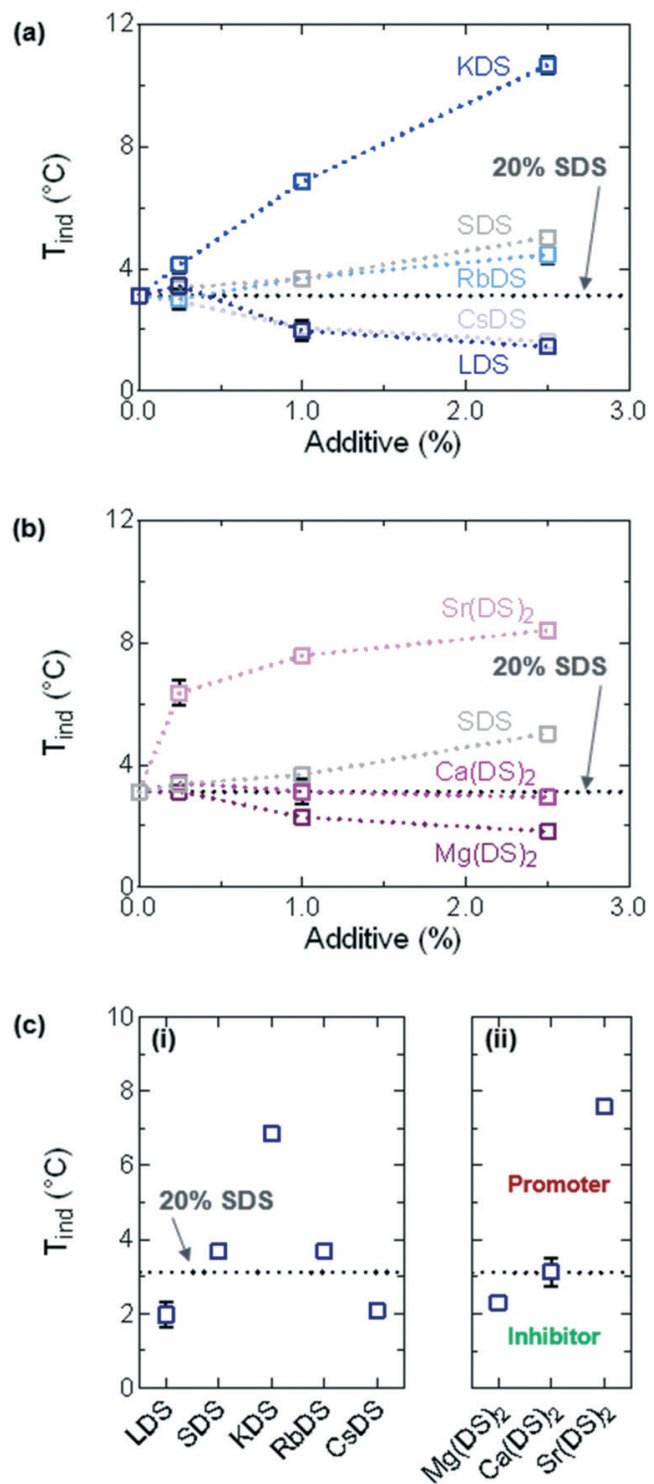

Fig. 3 Induction temperatures ( $\left.T_{\text {ind }}\right)$ extracted from DSC data for $20 \%$ SDS- $\mathrm{H}_{2} \mathrm{O}$ solutions containing additives (MDS), cooled at $\phi=0.5^{\circ} \mathrm{C}$ $\mathrm{min}^{-1}$. For $0.25-2.5 \%$ MDS (a) group 1 and (b) group 2 counterions. (c) For $1 \%$ MDS solutions (i) group 1 and (ii) group 2. The horizontal, dotted line represents the reference solution $20 \%$ SDS- $\mathrm{H}_{2} \mathrm{O}$.

tors, promoters or having a minor effect on SDS crystallisation. SDS, KDS, RbDS and $\mathrm{Sr}(\mathrm{DS})_{2}$ act as promoters - $2.5 \%$ KDS and $\operatorname{Sr}(\mathrm{DS})_{2}$ exert the strongest effect as they bring $T_{\text {ind }}$ up by $\approx 7.5$ and $\approx 5.3{ }^{\circ} \mathrm{C}$ respectively against the $20 \%$ reference or $\approx 5.6$ and $\approx 3.4{ }^{\circ} \mathrm{C}$ against SDS. RbDS and SDS have smaller effects against the $20 \%$ reference, increasing $T_{\text {ind }}$ by $\approx 1.6{ }^{\circ} \mathrm{C}$ for $2.5 \%$. Only $\mathrm{Ca}(\mathrm{DS})_{2}$ has a minor effect against the $20 \%$ reference. The remaining three additives LDS, CsDS and $\mathrm{Mg}(\mathrm{DS})_{2}$ inhibit crystallisation, $\approx 1.6^{\circ} \mathrm{C}$ for $2.5 \%$ LDS and CsDS and $\approx 1.3{ }^{\circ} \mathrm{C}$ for $2.5 \% \mathrm{Mg}(\mathrm{DS})_{2}$.

A clear concentration dependence is noted with additive addition, with all the additives (except $\mathrm{Ca}(\mathrm{DS})_{2}$ ) exerting stronger effects as promoters or inhibitors with increasing concentration. Discussed later, this is ascribed to lower and 
higher supersaturation for some of the inhibitors and promoters respectively and steric hinderance during incorporation of certain additives into the crystal structure, with all three parameters affected by concentration.

Overall the promoters have a stronger effect on $T_{\text {ind }}$ compared to the inhibitors which can be understood based on the system's MSZW. Discussed in further detail below the MSZW is already large at $\approx 16.0^{\circ} \mathrm{C}$. Suppressing the labile region even further is therefore likely to be problematic as the system is already very supercooled at this point and would have a high tendency to crystallise. This is supported by the fact that the decrease in $T_{\text {ind }}$ values shows signs of levelling off with increasing additive concentration for LDS and CsDS, whereas for the other additives the trend is almost linear.

In Fig. 3(c) $T_{\text {ind }}$ has been plotted for the $1 \%$ solutions. For group 1 given in Fig. 3(ci), with increasing ionic radius $T_{\text {ind }}$ initially follows the order LDS $<$ SDS $<$ KDS before decreasing in the same manner KDS $>$ RbDS $>$ CsDS. This trend could be caused by a variety of reasons including changes in the solubility, the coverage of the impurities, the driving force and other factors which will be discussed in turn below.

Solubility plays a key role in crystallisation which is both a temperature and concentration dependent parameter; ${ }^{24}$ for the analysis of the trends in Fig. 3(c) the focus is largely on temperature as the concentration has been fixed. With decreasing temperature the solubility also decreases; eventually the labile region of the saturation diagram is reached from which $T_{\text {ind }}$ values can be extracted. It is anticipated that additives such as KDS, (SDS) and RbDS reduce the solubility of the solution across all temperatures thereby acting as seed crystals or promoters of crystallisation, ${ }^{18,20}$ whilst LDS and CsDS may shift the supersolubility curve (but not the solubility curve), in turn reducing $T_{\text {ind }}$ and inhibiting crystallisation. Of note is that the exact trend observed here has been reported for the Krafft point of another group of surfactants, perfluorooctanic acid salts $(\mathrm{Li}<\mathrm{Na}<\mathrm{K}>\mathrm{Rb}>\mathrm{Cs}),{ }^{55}$ which was also rationalised based on the differing solubilities of the alkali metal salts.

The melting point trend for the group 1 dodecyl sulfate salts (the pure systems) supports this to an extent as it bears strong similarities to this mixed system: LDS $<$ SDS $\ll$ KDS $\approx$ RbDS $>\operatorname{CsDS}^{37,47,53,54}$ with the last two points proving slightly anomalous compared to what was observed here. From this it could be argued that the additives affect the solubility curve on the saturation diagram, however the melting point data for this system shows that the values are largely unaffected, discussed further in the DSC: heating ramps section. The driving force of these additives is thus expected to be kinetic as opposed to thermodynamic. Consequently, promoters act to seed crystallisation whilst inhibitors either lower the supersaturation or impact incorporation of the units into the crystal structure; the latter is discussed below.

The coverage of the impurities is likely to depend on the counterion size which is affected by two factors - the hydration state ${ }^{51,52}$ decreases going down the group due to increased charge screening, however the ionic radius of the cat- ion also increases. The large steric size at both extremes potentially slows down incorporation into the crystal structure, in turn causing the reduction in $T_{\text {ind }}$ values at either end of the group. Of note is that this hindrance does not always affect incorporation of the additives into the crystal structure, which is discussed further in the ICP-OES section. LDS for instance was the only additive not to be incorporated into the crystal, therefore its effect on $T_{\text {ind }}$ is likely to be due to a change in supersaturation, whilst CsDS is more likely to slow down the kinetics of incorporation due to size issues.

For group $2 T_{\text {ind }}$ increases going down the group, shown in Fig. 3(cii). $\mathrm{Mg}(\mathrm{DS})_{2}$ acts as an inhibitor whilst $\mathrm{Ca}(\mathrm{DS})_{2}$ has little effect on $T_{\text {ind }}$ when compared to the reference. Similar points discussed for the group 1 additives apply in terms of solubility, with $\mathrm{Mg}(\mathrm{DS})_{2}$ potentially shifting the supersolubility curve whilst $\mathrm{Ca}(\mathrm{DS})_{2}$ had little effect. Again a kinetic driving force is expected as the melting points for all three remain relatively unchanged. $\mathrm{Sr}(\mathrm{DS})_{2}$ was the only promoter in group 2 which exhibited significant insolubility across the investigated temperature range, thereby potentially acting as a seed crystal and increasing the value of $T_{\text {ind }}$. The limited solubility of $\operatorname{Sr}(\mathrm{DS})_{2}$ is discussed more in the heating ramps section.

According to the Hofmeister series these cations are the most kosmotropic in the order $\mathrm{Mg}^{2+}>\mathrm{Ca}^{2+}, 51,52$ more so than the group 1 cations due to their divalent charge. It is therefore also plausible that the inhibitory effect of $\mathrm{Mg}^{2+}$ is due to steric bulk as opposed to a shift in supersolubility.

\section{DSC: heating ramps}

DSC traces for the 1\% MDS solutions are presented in Fig. 4(a) and (b) for the group 1 and 2 based additives respectively; the $20 \%$ SDS $-\mathrm{H}_{2} \mathrm{O}$ reference solution is the dotted, black line. From these curves it is apparent that all follow the same generic pattern, displaying a broad transition indicative of the crystalline phase melting down to a micellar solution. The features on the left of the peak are interpreted as the interconversion to the more stable dihydrate crystal. The melting point is taken as the peak minimum from the steepest slope which is plotted in Fig. 5(a), also where full dissolution was observed via OM.

For the majority of group 1 in Fig. 5(ai) the melting points display a minor shift compared to $20 \%$ SDS- $\mathrm{H}_{2} \mathrm{O}$, with RbDS deviating the most at $0.6 \pm 0.3{ }^{\circ} \mathrm{C}$. For group 2 in Fig. 5(aii) $T_{0}$ values shift more from the reference, although this is still only $0.9 \pm 0.1{ }^{\circ} \mathrm{C}$ in the largest case $-\operatorname{Sr}(\mathrm{DS})_{2}$. Overall for both groups no significant impact on $T_{0}$ is noted with the addition of the additives. This suggests that their effect is predominantly kinetic as opposed to thermodynamic, with the counterions hindering or promoting crystal nucleation or growth.

While seven of the eight solutions containing additives melted to form a micellar solution at $T_{0}, \operatorname{Sr}(\mathrm{DS})_{2}$ solutions contained a residual precipitate with a partially crystalline texture. To determine the temperature at which this 


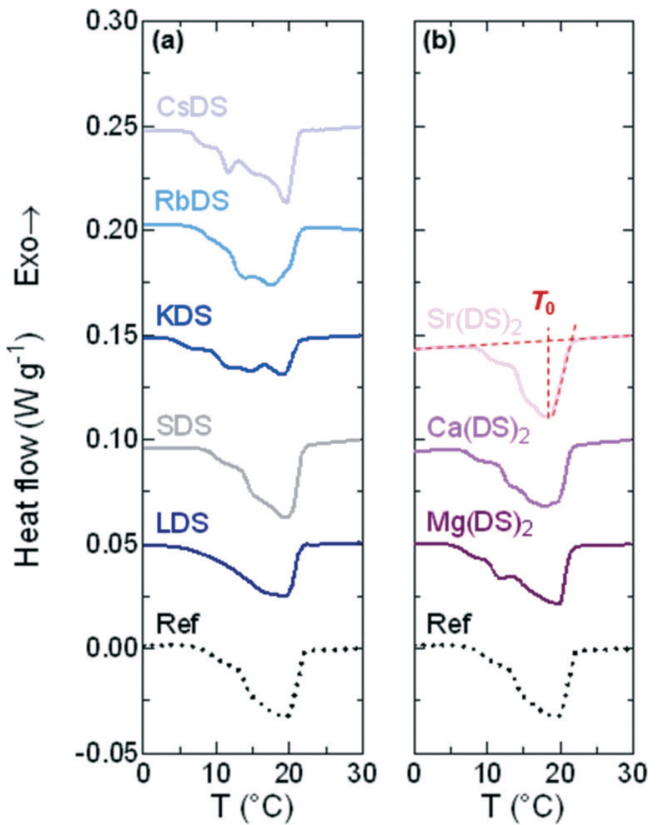

Fig. 4 DSC traces $20 \%$ SDS solutions spiked with $1 \%$ additives (MDS). These were heated linearly at $\phi=0.5{ }^{\circ} \mathrm{C} \mathrm{min}^{-1}$ from the crystalline phase for (a) group 1 and (b) group 2 traces. The dotted line represents the reference solution $20 \%$ SDS- $\mathrm{H}_{2} \mathrm{O}$, with traces shifted in increments of $0.05 \mathrm{~W} \mathrm{~g}^{-1}$ for clarity.

precipitate dissolved for $1 \% \operatorname{Sr}(\mathrm{DS})_{2}$ the solution's turbidity was measured via DLS. Described further in the experimental section the mean count rate of the solution was measured every $0.5{ }^{\circ} \mathrm{C}$ from 20 to $37^{\circ} \mathrm{C}$. From this it was determined that the precipitate fully dissolves by $33 \pm 0.5{ }^{\circ} \mathrm{C}$ when the curve reached a plateau, ESI $\uparrow$ (Fig. S1). The other $1 \%$ solutions were also measured for comparison at $1{ }^{\circ} \mathrm{C}$ intervals, confirming that precipitates were not present in solution after melting was complete as the profiles were flat.

The MSZW is the difference between the saturation temperature $\left(T_{0}\right)$ and the temperature at which crystallisation is detected $\left(T_{\text {ind }}\right)$ for a given cooling rate: $\mathrm{MSZW}=T_{0}-T_{\text {ind. }}{ }^{56-58}$ The MSZW for $20 \%$ SDS- $\mathrm{H}_{2} \mathrm{O}$ at $\phi=0.5{ }^{\circ} \mathrm{C} \mathrm{min}{ }^{-1}$ is already relatively large at $16.0 \pm 0.1{ }^{\circ} \mathrm{C}$ compared to other systems (such as simple inorganic salts), ${ }^{59}$ possibly due to the rearrangement which occurs when these micellar solutions crystallise to form a lamellar structure.

For the most part the MSZW in Fig. 5(b) is the reverse of $T_{\text {ind }}$ because the melting point remains relatively unchanged. LDS, CsDS and $\mathrm{Mg}(\mathrm{DS})_{2}$ widen the MSZW by $\approx 1.3^{\circ} \mathrm{C}$, increasing the uncertainty window of crystallisation. SDS, RbDS and $\mathrm{Ca}(\mathrm{DS})_{2}$ have a smaller effect as their impact on $T_{\text {ind }}$ was lower, on average changing the MSZW by $0.1 \pm 0.5^{\circ} \mathrm{C}$. KDS and $\operatorname{Sr}(\mathrm{DS})_{2}$ lower the MSZW by $\approx 3.9$ and $\approx 5.3{ }^{\circ} \mathrm{C}$ respectively by making the solution more prone to crystallisation, thereby reducing this uncertainty window. As mentioned previously the MSZW is already large and the system is very supercooled. Further widening of the MSZW is thus more challenging than suppression, therefore it is expected that additives will promote as opposed to inhibit crystallisation far more readily. These effects are significant and are induced by a relatively small quantity of additive (1\%). These can easily enter the system during manufacturing, for example, if non-deionised water is used due to preferential $\mathrm{Na}$ exchange.

\section{Optical microscopy: morphologies}

The crystallisation of micellar SDS- $\mathrm{H}_{2} \mathrm{O}$ solutions was previously found to yield a range of morphologies from platelets through to needles depending on the temperature ${ }^{27}$ and cooling rate. ${ }^{47}$ The impact of linear cooling conditions is useful to parameterise as most formulations are likely to experience temperature variations under practically relevant conditions. With the presence of additives however $T_{\text {ind }}$ occurs at a variety of temperatures for a given rate, as shown above. Different morphologies and kinetics would therefore be expected, making it challenging to determine whether these are caused by the additive addition or the temperature at which crystallisation occurred. The approach chosen via OM was therefore isothermal holds at $T=6^{\circ} \mathrm{C}$, which also provided additional kinetic data by employing a different type of temperature profile.

Images of the representative crystal morphology observed for the 1\% additives are given in Fig. 6(a). Whilst the reference solution yielded an octagonal platelet at $T=6{ }^{\circ} \mathrm{C}$ a
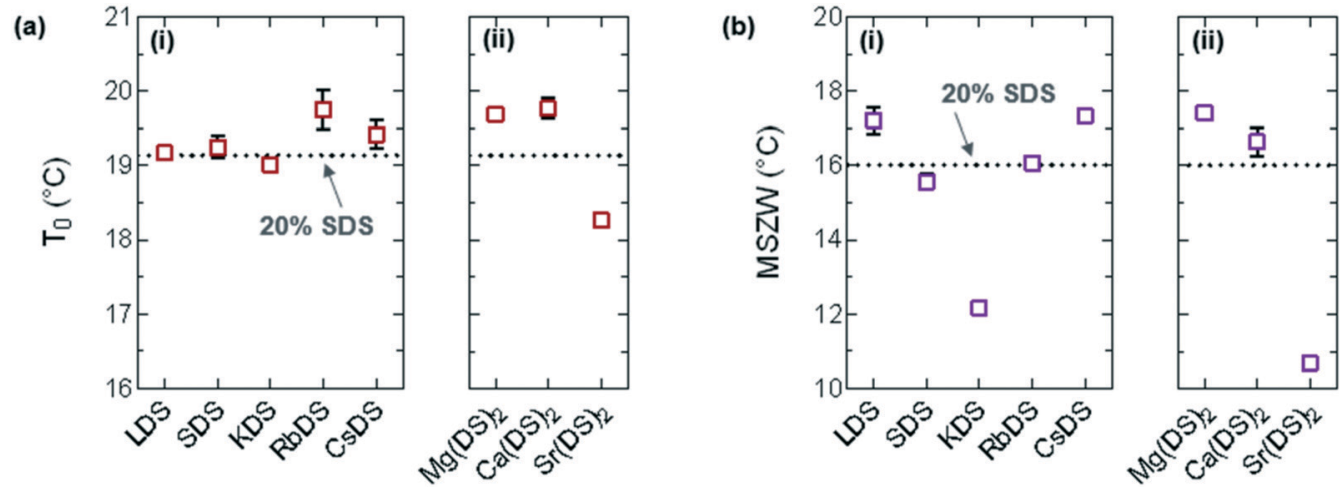

Fig. 5 (a) Melting point $\left(T_{0}\right)$ taken as the minimum from the steepest slope of the heating curve and (b) MSZW $=T_{0}-T_{\text {ind }}$ for (i) group 1 and (ii) group 2. 
(a)
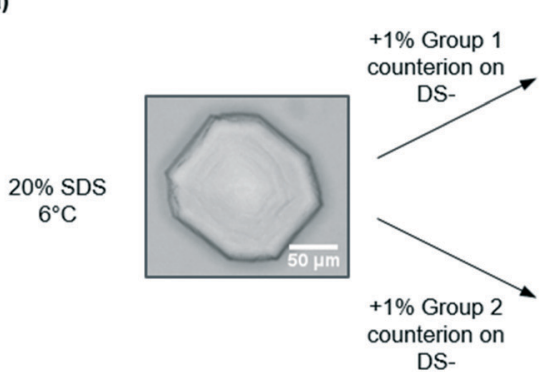

(b)
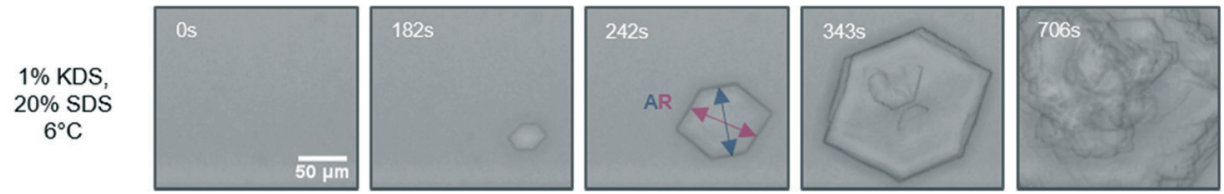

Fig. 6 (a) Optical microscopy images depicting the representative crystal morphology for each $20 \%$ SDS- $\mathrm{H}_{2} \mathrm{O}$ solution doped with $1 \%$ additive for the group 1 and 2 based counterions. Samples were quenched to $T=6{ }^{\circ} \mathrm{C}$ and held isothermally until crystallisation was complete. (b) The development of crystallisation over time for $1 \%$ KDS, from which the induction time $\left(t_{\text {ind }}\right)$ and the aspect ratio (AR) were determined. All scale bars are $50 \mu \mathrm{m}$.

variety of different morphologies are observed with additives, which are generically characterised as platelets. 1\% KDS and RbDS give hexagonal and diamond shaped crystals respectively, both of which differ from the reference due to increasing omission of sides. These crystals grow via structural evolution, ${ }^{60}$ possibly due to the additive binding to the crystal face which inhibits their growth. LDS, SDS and CsDS retained the octagonal form, with LDS and SDS comparable to the reference solution. In contrast four of the faces for CsDS are truncated, again attributed to additive inhibition.

For group 2 the changes are more prominent as shown in Fig. 6(a). $1 \% \mathrm{Mg}(\mathrm{DS})_{2}$ and $\mathrm{Ca}(\mathrm{DS})_{2}$ give oval shaped crystals which develop features over time. With $1 \% \mathrm{Mg}(\mathrm{DS})_{2}$ rapid growth occurs from one or more sides of the crystal, whereas for $\mathrm{Ca}(\mathrm{DS})_{2}$ darker spots arise, as shown in ESI $\dagger$ (Fig. S2a and b). Although $\mathrm{Ca}(\mathrm{DS})_{2}$ has a minimal impact on $T_{\text {ind }}$, complementary microscopy data establishes its effect as an additive. $\mathrm{Sr}(\mathrm{DS})_{2}$ exhibits a considerable change in morphology over the crystallisation period - whilst this briefly begins as an oval platelet, rapid growth ensues from the sides to give large, thick needles, shown in Fig. 6(a) and ESI $\uparrow$ (Fig. S2c). Overall most of the morphologies observed here differ to those formed during the isothermal and linear cooling studies of SDS- $\mathrm{H}_{2} \mathrm{O},{ }^{27,47}$ therefore are ascribed to the additives.

\section{Optical microscopy: integration and crystallisation kinetics}

ICP-OES was combined with the microscopy results to understand whether there was a relationship between the two data sets, specifically whether each additive was integrated into the crystal, directed crystallisation or had no effect on the morphology. ICP-OES is not a standard method to analyse whether an additive is integrated into a crystal. Traditional techniques focus on XRD of the crystal itself or washings from the surface of the crystal to analyse whether an additive is completely or surface-integrated respectively. In this instance this novel approach was preferred due to its ease and high sensitivity. The supernatant was selected over the crystal itself as residual supernatant on the crystal's surface can skew the results whilst washing this off potentially removes any surface-integrated additive, particularly problematic for this system due to the small size of the crystals.

The percentage of both $\mathrm{Na}$ and the counterion ("M") were determined for the starting solution (which served as a second set of references in addition to the standards) and the supernatant of the crystallised solutions. The concentration (ppm) of ions in the original solution and supernatant after dilution were converted to percentages relative to $20 \%$ SDS or $1 \%$ additive for ease of interpretation - for example the $\mathrm{Na}$ content in the starting solution is $1.59 \%$ or $15.9 \mathrm{ppm}$. These calculations along with the dilutions are provided in the ESI. $\dagger$

In Fig. 7(a) the dotted line at the top represents the normalised $\mathrm{Na}$ content pre-crystallisation, whilst the square symbols are the normalised $\mathrm{Na}$ in the supernatant. From these results it is noted that almost all the $\mathrm{Na}$ is integrated in the crystal across the additives, with the marginally higher value for $\mathrm{Li} \approx 1.36 \%$ compared to an average of $\approx 0.46 \%$ (maximum: $20 \%$ ) considered to be within experimental error. For Fig. 7(b) the dotted line and symbols are for the additive, again normalised. In most cases almost no additive remains in the solution which correlates to the (mostly) pronounced morphological changes observed in Fig. 6(a). The exceptions to this are LDS which shows little sign of integration as 0.87 $\pm 0.10 \%$ (maximum: $1.00 \%$ ) $\mathrm{Li}$ remains in the supernatant and additional SDS, both of which retain the octagonal morphology with similar dimensions to the reference solution.

The induction times $\left(t_{\text {ind }}\right)$ taken from optical microscopy have been plotted in Fig. 7(c); the values represent upper 
(a)

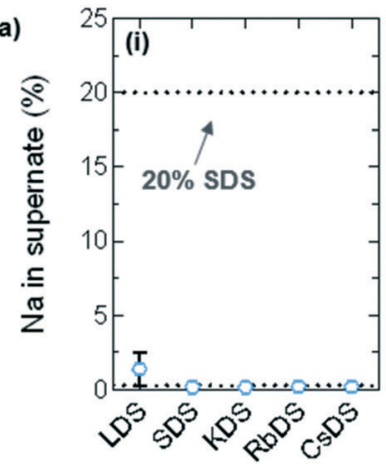

(c)
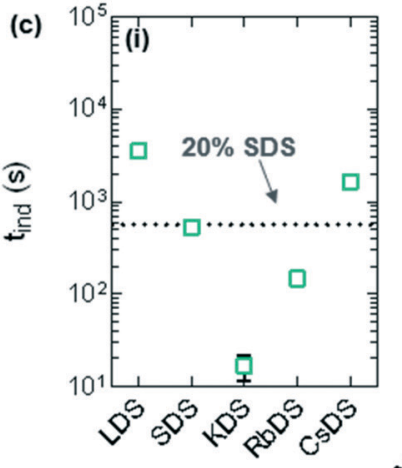
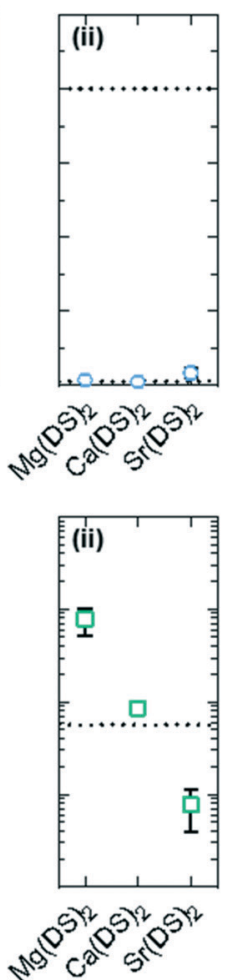
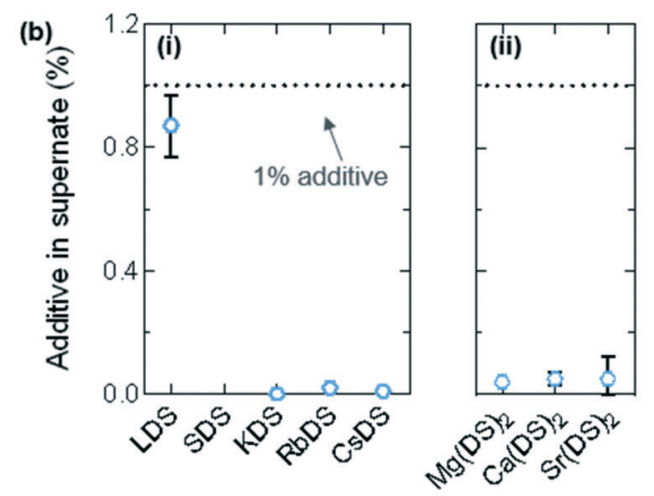

(d)

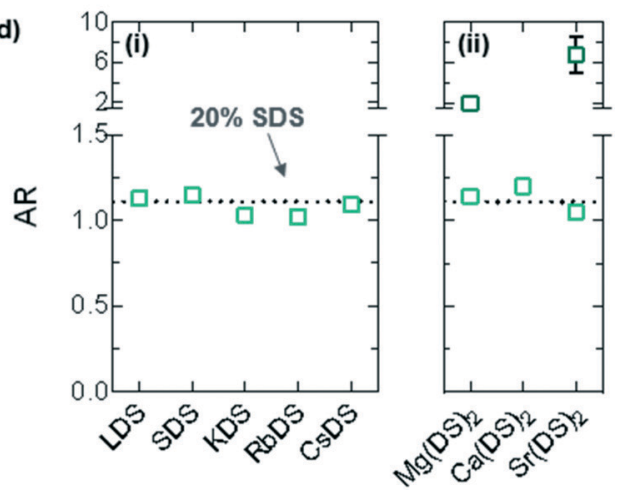

Fig. 7 Determination of the ion concentration in the supernatant using ICP-OES after crystallisation was complete and crystallisation kinetics from OM. (a) Percentage of Na normalised to the SDS concentration (20\% - the dotted line at the top) for (i) group 1 and (ii) group 2 . (b) Percentage of the additive's counterion (M) normalised to its (MDS) concentration (1\% - the dotted line at the top) for (i) group 1 and (ii) group 2 . This has been omitted for SDS as the $1 \%$ cannot be distinguished from the $20 \%$. (c) Induction times ( $\left.t_{\text {ind }}\right)$ from OM data at $T=6{ }^{\circ} \mathrm{C}$ for (i) group 1 and (ii) group 2 . (d) Aspect ratio (AR) for the crystals in (i) group 1 and (ii) group 2; the darker points $>1.5$ for $\mathrm{mg}(\mathrm{DS})_{2}$ and $\operatorname{Sr}(\mathrm{DS})_{2}$ include the dimensions of any protrusions which formed during crystal growth.

limits given the finite spatial resolution of microscopy. ${ }^{5}$ These are the reverse of the $T_{\text {ind }}$ values noted in Fig. 3(c), specifically the additives that exhibited a low $T_{\text {ind }}$ in the linear cooling experiments have a correspondingly long $t_{\text {ind }}$ value due to increased metastability. $\mathrm{Mg}(\mathrm{DS})_{2}$ for example has a $t_{\text {ind }}$ $\approx 7.8 \pm 2.5 \times 10^{3} \mathrm{~s}$ and $T_{\text {ind }} \approx 2.3{ }^{\circ} \mathrm{C}$, whereas KDS has a $t_{\text {ind }}$ $\approx 17 \pm 5 \mathrm{~s}$ and $T_{\text {ind }} \approx 6.8^{\circ} \mathrm{C}$.

The evolution of the crystal morphology with time was used to determine the aspect ratio (AR) by averaging the length to width ratio ${ }^{61}$ across the images. This is shown in Fig. 6(b) for KDS; the last image in the series only shows surface features as the crystal has outgrown the illustrative area. $1 \%$ LDS, SDS and CsDS had the lowest impact on the AR, averaging $1.12 \pm 0.03$ compared to the reference AR of $1.11 \pm 0.01$. $1 \%$ KDS and RbDS crystals visibly grew smaller and had a notably reduced AR of $1.03 \pm 0.01$ and $1.02 \pm 0.01$ respectively. Although the cooling rate is often changed to produce smaller size crystals ${ }^{62}$ to improve filtration or dissolution properties here the same effect has been achieved with additives.

For group 2 the oval-shaped $\mathrm{Ca}(\mathrm{DS})_{2}$ crystal exhibited the lowest increase in AR to $1.20 \pm 0.03$, which was the only one to have any protrusions. $\mathrm{Mg}(\mathrm{DS})_{2}$ began with this oval base that has an AR of $1.14 \pm 0.04$ however the protrusions brought this up to $1.90 \pm 0.08$, as noted by the darker points in Fig. 7 (d). With $\operatorname{Sr}(\mathrm{DS})_{2}$ the thick needles bring its AR up con- siderably to $6.74 \pm 1.74$. Needles are generally considered to be an undesirable morphology due to their poor flow properties. ${ }^{63,64}$

While the group 1 counterions demonstrated small changes in the AR of $20 \%$ SDS- $\mathrm{H}_{2} \mathrm{O}$ crystals, the group 2 counterions significantly increased this. Depending on the use, a lower AR may be preferred to reduce compact layer

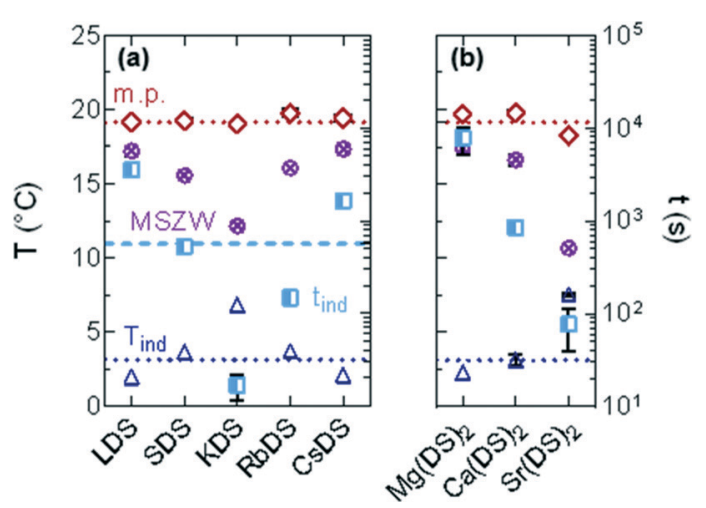

Fig. 8 Summary of the trends observed when $1 \%$ of a structurally similar SDS additive (MDS) was added to $20 \%$ SDS- $\mathrm{H}_{2} \mathrm{O}$. Left axis: $T_{\text {ind }}$ (dark blue), melting point (red) and MSZW (purple); right: $t_{\text {ind }}$ (light blue) for the counterions in (a) group 1 and (b) group 2. The dotted lines are the values for $20 \%$ SDS- $\mathrm{H}_{2} \mathrm{O}$. 
formation at the bottom of filtration media or to alter flow properties. Overall small quantities of structurally similar SDS additives on which the counterion is varied have notable kinetic and morphological effects on SDS- $\mathrm{H}_{2} \mathrm{O}$ crystallisation, as summarised in Fig. 8.

\section{Conclusions}

The effect of structurally similar additives on the crystallisation kinetics and morphologies of the ubiquitous SDS- $\mathrm{H}_{2} \mathrm{O}$ micellar system was investigated under isothermal and linear cooling conditions. Seven different counterions from groups 1 and 2 of the periodic table were chosen to replace the sodium on SDS to give the following additives: LDS, (SDS), KDS, RbDS, CsDS, $\mathrm{Mg}(\mathrm{DS})_{2}, \mathrm{Ca}(\mathrm{DS})_{2}$ and $\mathrm{Sr}(\mathrm{DS})_{2}$, which were added to a reference solution of $20 \%$ SDS- $\mathrm{H}_{2} \mathrm{O}$. DSC at a fixed cooling rate $\phi=0.5{ }^{\circ} \mathrm{C} \min ^{-1}$ for $0.25,1.0$ and $2.5 \%$ additive showed that (additional SDS), KDS, RbDS, $\operatorname{Sr}(\mathrm{DS})_{2}$ promoted crystallisation, $\mathrm{Ca}(\mathrm{DS})_{2}$ had a minor effect and LDS, CsDS, $\mathrm{Mg}(\mathrm{DS})_{2}$ inhibited crystallisation. This was rationalised based on available solubility data, the counterion size and hydration state. The changes in melting point were found to be minimal suggesting that the additives had a kinetic as opposed to a thermodynamic effect. As such the MSZWs were essentially the reverse of $T_{\text {ind }}$. Complementary data from DLS suggests that all the additives except $\operatorname{Sr}(\mathrm{DS})_{2}$ undergo transitions from the crystalline phase to give a micellar solution; this additive requires higher temperatures to dissolve a residual precipitate.

Optical microscopy for the $1 \%$ solutions shows that most additives had an impact on the morphology at $T=6^{\circ} \mathrm{C}$. For group 1 RbDS and KDS gave hexagonal and diamond shaped crystals which exhibited a lower AR than the reference, often considered more desirable as they are less "sheet-like". LDS, SDS and CsDS however retained the reference morphology. The basis of group 2 are oval-shaped crystals which exhibit protrusions for $\mathrm{Mg}(\mathrm{DS})_{2}$ and the dominance of thick, platelet-type needles originating from centre for $\operatorname{Sr}(\mathrm{DS})_{2}$. These needles had the largest $\mathrm{AR}$, a potential drawback when it comes to processing and dissolution. OM data was supported by ICP-OES which indicated that the morphological changes were caused by integration of the additives into the crystal. Lastly, although many of the $T_{\text {ind }}$ values were lower than $6^{\circ} \mathrm{C}$ during the cooling ramp studies, $t_{\text {ind }}$ values from OM were the reverse of $T_{\text {ind }}$ due to increased metastability.

Overall small (0.25 to $2.5 \%)$ quantities of structurally similar additives had a relatively potent effect on $\mathrm{SDS}-\mathrm{H}_{2} \mathrm{O}$ crystallisation. These additives can easily form if nondeionised water enters the system, for instance, as the $\mathrm{Na}$ on SDS is preferentially exchanged by multiple cations from group 1 and 2. Additives also enable tailoring of both the crystal morphology and the potential to increase or decrease the solution's stability below the micellar-to-crystalline phase boundary.

\section{Conflicts of interest}

There are no conflicts to declare.

\section{Acknowledgements}

We thank the Engineering and Physical Sciences Research Council (EPSRC) Institute of Chemical Biology-CDT (EP/ L015498/1) and The Procter \& Gamble Company (P\&G) for a PhD studentship for RMM. We are grateful to Ms. Patricia Carry for use of the DSC and ICP-OES, and Dr. Andreas Poulos for assistance with DLS measurements.

\section{Notes and references}

1 A. E. Hargreaves, Chemical Formulation: An Overview of Surfactant Based Preparations Used in Everyday Life, Royal Society of Chemistry, Cambridge, U.K., 2003.

2 M. Patel, J. Ind. Ecol., 2004, 7, 47-62.

3 K. Hill, Pure Appl. Chem., 2000, 72, 1255-1264.

4 I. Johansson and M. Svensson, Curr. Opin. Colloid Interface Sci., 2001, 6, 178-188.

5 M. A. Lovette, A. R. Browning, D. W. Griffin, J. P. Sizemore, R. C. Snyder and M. F. Doherty, Ind. Eng. Chem. Res., 2008, 47, 9812-9833.

6 K. Fontell, Mol. Cryst. Liq. Cryst., 1981, 63, 59-82.

7 K. J. Mysels, Langmuir, 1986, 2, 423-428.

8 P. H. Elworthy and K. J. Mysels, J. Colloid Interface Sci., 1966, 21, 331-347.

9 I. Weissbuch, R. Popovitz-Biro, M. Lahav and L. Leiserowitz, Acta Crystallogr., Sect. B: Struct. Sci., 1995, 51, 115-148.

10 N. Rodríguez-Hornedo and D. Murphy, J. Pharm. Sci., 1999, 88, 651-660.

11 J. Garside, A. Mersmann and J. Nyvlt, Measurement of Crystal Growth and Nucleation Rates, Institution of Chemical Engineers, Rugby, 2nd edn, 2002.

12 A. Van Hook, Crystallization: Theory and Practice, Reinhold Pub. Corp., New York, 1961.

13 I. Sunagawa, Crystals: Growth, Morphology and Perfection, Cambridge University Press, Cambridge, 2005.

14 D. Mangin, F. Puel and S. Veesler, Org. Process Res. Dev., 2009, 13, 1241-1253.

15 D. Lechuga-Ballesteros and N. Rodríguez-Hornedo, Int. J. Pharm., 1955, 115, 151-160.

16 C. Gu, K. Chatterjee, V. Young and D. J. W. Grant, J. Cryst. Growth, 2002, 235, 471-481.

17 V. Fischer, K. Landfester and R. Muñoz-Espí, Cryst. Growth Des., 2011, 11, 1880-1890.

18 K. Sangwal, Additives and Crystallization Processes: From Fundamentals to Applications, John Wiley, England, 2007.

19 M. Kind, Chem. Eng. Process., 1999, 38, 405-410.

20 R. J. Davey, S. L. M. Schroeder and J. H. ter Horst, Angew. Chem., Int. Ed., 2013, 52, 2166-2179.

21 M. J. Rosen, Surfactants and Interfacial Phenomena, John Wiley, New Jersey, 3rd edn, 2004. 
22 E. Smulders, W. von Rybinsk and A. Nordskog, Ullmann's Encyclopedia of Industrial Chemistry, Wiley-VCH Verlag GmbH \& Co. KGaA, Weinheim, Germany, 2011.

23 J. Bibette, F. L. Calderon and P. Poulin, Rep. Prog. Phys., 1999, 62, 969-1033.

24 J.-C. Baret, Lab Chip, 2012, 12, 422-433.

25 P. Kékicheff, C. Grabielle-Madelmont and M. Ollivon, J. Colloid Interface Sci., 1989, 131, 112-132.

26 P. Kékicheff, J. Colloid Interface Sci., 1989, 131, 133-152.

27 R. M. Miller, A. S. Poulos, E. S. J. Robles, N. J. Brooks, O. Ces and J. T. Cabral, Cryst. Growth Des., 2016, 16, 3379-3388.

28 V. M. Coiro, F. Mazza and G. Pochetti, Acta Crystallogr., Sect. C: Cryst. Struct. Commun., 1986, 42, 991-995.

29 V. M. Coiro, M. Manigrasso, F. Mazza and G. Pochetti, Acta Crystallogr., Sect. C: Cryst. Struct. Commun., 1987, 43, 850-854.

30 S. Sundell, Acta Chem. Scand., Ser. A, 1977, 31, 799-807.

31 L. A. Smith, R. B. Hammond, K. J. Roberts, D. Machin and G. McLeod, J. Mol. Struct., 2000, 554, 173-182.

32 F. F. J. Rawlings and E. C. Lingafelter, J. Am. Chem. Soc., 1950, 72, 1852.

33 F. F. J. Rawlings and E. C. Lingafelter, J. Am. Chem. Soc., 1955, 77, 870-872.

34 V. M. Coiro and F. Mazza, Acta Crystallogr., Sect. C: Cryst. Struct. Commun., 1989, 45, 1132-1136.

35 V. M. Coiro and F. Mazza, Acta Crystallogr., Sect. C: Cryst. Struct. Commun., 1991, 47, 1169-1173.

36 R. P. Sperline, Langmuir, 1997, 13, 3715-3726.

37 L. A. Smith, K. J. Roberts, D. Machin and G. McLeod, J. Cryst. Growth, 2001, 226, 158-167.

38 N. Rodríguez-Hornedo and D. Murphy, J. Pharm. Sci., 2004, 93, 449-460.

39 X.-Y. Sun, J.-M. Ouyang and M. Xu, CrystEngComm, 2016, 18, 5463-5473.

40 S. Kumar, G. Chawla and A. K. Bansal, Yakugaku Zasshi, 2008, 128, 281-289.

41 G. Liu, J. Liu, X. Tao, D.-S. Li and Q. Zhang, Inorg. Chem. Front., 2016, 3, 1388-1392.

42 H. Wei, Q. Shen, Y. Zhao, Y. Zhou, D. Wang and D. Xu, J. Cryst. Growth, 2005, 279, 439-446.
43 M. H. H. Mahmoud, M. M. Rashad, I. A. Ibrahim and E. A. Abdel-Aal, J. Colloid Interface Sci., 2004, 270, 99-105.

44 E. Summerton, G. Zimbitas, M. Britton and S. Bakalis, J. Cryst. Growth, 2016, 455, 111-116.

45 M. Muramatsu and M. Inoue, J. Colloid Interface Sci., 1976, 55, 80-84.

46 D. Bethell, R. E. Fessey, E. Namwindwa and D. W. Roberts, J. Chem. Soc., Perkin Trans. 2, 2001, 1489-1495.

47 R. M. Miller, O. Ces, N. J. Brooks, E. S. J. Robles and J. T. Cabral, Cryst. Growth Des., 2017, 17, 2428-2437.

48 S. Miyamoto, Bull. Chem. Soc. Jpn., 1960, 33, 371-375.

49 Y. Moroi, K. Motomura and R. Matuura, J. Colloid Interface Sci., 1974, 46, 111-117.

50 A. Jeziorny, Polymer, 1978, 19, 1142-1144.

51 F. Hofmeister, Arch. Exp. Pathol. Pharmakol., 1888, 24, 247-260.

52 W. Kunz, J. Henle and B. W. Ninham, Curr. Opin. Colloid Interface Sci., 2004, 9, 19-37.

53 K. Tamori, K. Esumi and K. Meguro, J. Colloid Interface Sci., 1991, 142, 236-243.

54 A. Vagias, W. L. Suszynski, H. T. Davis and A. V. McCormick, J. Chem. Eng. Data, 2017, 62, 1623-1627.

55 H. Nakayama, Bull. Chem. Soc. Jpn., 1967, 40, 1592-1595.

56 N. Lyczko, F. Espitalier, O. Louisnard and J. Schwartzentruber, Chem. Eng. J., 2002, 86, 233-241.

57 K. Sangwal, Cryst. Growth Des., 2009, 9, 942-950.

58 J. W. Mullin, Crystallization, Butterworth-Heinemann, Oxford, 4th edn, 2001.

59 J. Nývlt, R. Rychlý, J. Gottfried and J. Wurzelová, J. Cryst. Growth, 1970, 6, 151-162.

60 Y. Zhang, J. P. Sizemore and M. F. Doherty, AIChE J., 2006, 52, 1906-1915.

61 G. Yang, N. Kubota, Z. Sha, M. Louhi-Kultanen and J. Wang, Cryst. Growth Des., 2006, 6, 2799-2803.

62 C. Wibowo and K. M. Ng, AIChE J., 2001, 47, 2746-2767.

63 N. Variankaval, A. S. Cote and M. F. Doherty, AIChE J., 2008, 54, 1682-1688.

64 W. M. L. Wood, Powder Technol., 2001, 121, 53-59. 